26. Y.S. Liu, Research on key techniques and implementation of highspeed optical short-pulse sources and ultrawide-bandwidth optical oscilloscope, PhD Dissertation, Xi'an Institute of Optics and Precision Mechanics, Chinese Academy of Sciences, Xi'an, China, 2008.

(C) 2011 Wiley Periodicals, Inc.

\section{CAVITY-BACKED SLOT ARRAY ANTENNA IN SUBSTRATE-INTEGRATED WAVEGUIDE TECHNOLOGY}

\author{
Manuel J. Jiménez-Fernández, Roberto Torres-Sánchez, \\ and Pablo Otero \\ Department of Communications Engineering, University of Málaga, \\ Málaga 29071, Spain; Corresponding author: otero@ic.uma.es
}

Received 19 November 2010

ABSTRACT: A slot array placed on the face of a cavity realized in substrate-integrated waveguide technology is presented. Guidelines to design similar antennas are provided. The design has been done with the help of commercial software. The result is a low-cost, directive array antenna with a single feeding microstrip-line. (C) 2011 Wiley Periodicals, Inc. Microwave Opt Technol Lett 53:2105-2108, 2011; View this article online at wileyonlinelibrary.com. DOI 10.1002/mop.26165

Key words: cavity-backed antenna; SIW technology; slot array

\section{INTRODUCTION}

In the last years, the demand for low cost and high performance wireless communication systems has increased significantly. As a result, there is a growing need for antennas that allow efficient transmission and reception of information signals. In particular, printed slot array antennas are very convenient because of their attractive characteristics such as high directivity, conformability, easy integration with planar circuits, and simple feeding structure and low cost. These slot arrays have been widely studied [1-6].

In this article, a cavity-backed slot array based on the studies of Paoloni [2] is proposed. One of the most interesting features of the new design is the simplicity of the array feeding, which is implemented with a single microstrip-line coupled to the cavity through a slot in its backside. Ideally, there is a resonant monomode stationary wave in the cavity. In principle, the illumination of the array aperture can be designed by properly placing the radiating slots on the upper cavity wall, when known the field distribution within the cavity. In fact, with the application of this technique, one of the major issues in array antenna design (i.e., the routing of the beamforming network) is conveniently circumvented. For the present array, a uniform illumination is achieved by locating the slots at points, where the electric field has equal phase and maximum magnitude.

The cavity is made using the innovative substrate-integrated waveguide (SIW) technique, which was originally proposed for constructing conventional waveguides using printed-circuit technology [7, 8]. The use of SIW technique and the aperture coupled feeding line allow a full photolithographic manufacturing process. The principal aim of this work is to demonstrate the feasibility of the slot array in SIW technology with a planar feed. The lateral walls of the SIW cavity consist of linear arrays of metallic posts that short circuit upper and lower faces of the cavity. Besides, some other posts are strategically inserted in the cavity to filter unwanted resonant modes.

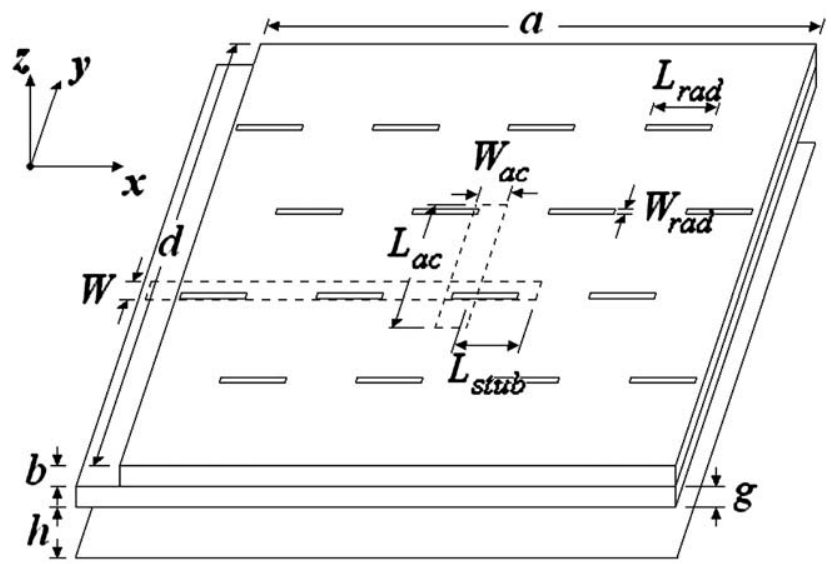

Figure 1 Antenna structure

\section{ANTENNA DESCRIPTION AND DESIGN}

A slot array printed on the upper face of a rectangular resonant cavity is proposed. A schematic view of the antenna structure is shown in Figure 1 . The array may have $N \times M$ equal radiating slots with dimensions $L_{\text {rad }} \times W_{\text {rad. }}$ The distances between slot centers along $x$ and $y$ axes are, respectively, $d_{x}$ and $d_{y}$. For the antenna to have proper directive radiation, the radiating slots must be fed in phase. Hence, they must be placed in alternate points of maximum electric field. The dimensions of the cavity are $a \times b \times d$; these dimensions determine the resonance frequencies of the modes within the cavity. The cavity is filled with a dielectric (the upper substrate) of permittivity $\varepsilon_{\mathrm{rc}}$ and loss tangent $(\tan \delta)_{\mathrm{c}}$. As said before, lateral walls are made with SIW technique, the distance between consecutive posts is $p_{\mathrm{p}}$, and the posts diameter is $d_{\mathrm{p}}$. The via holes' distance and diameter are designed according to Ref. 8. If lengths $a, b$, and $d$ are measured between posts centers, the effective dimensions of the cavity to calculate resonant frequencies are $a_{\text {eff }} \times b_{\text {eff }} \times d_{\text {eff. }}$. The effective lengths can be calculated as $a_{\text {eff }}=a-d_{\mathrm{p}}^{2} /\left(0.95 p_{\mathrm{p}}\right)$ and a similar equation for $d_{\mathrm{eff}}$. This approximation is valid for $p_{\mathrm{p}}<\lambda_{0} \sqrt{\varepsilon_{\mathrm{rc}}} / 2$ and $p_{\mathrm{p}}<4 d_{\mathrm{p}}$ [9]. Starting dimensions for the radiating slots are $L_{\mathrm{rad}}=\lambda_{\mathrm{g}} / 2$ and $W_{\text {rad }}=L_{\text {rad }} / 5$, where $\lambda_{\mathrm{g}}=\sqrt{2} \lambda_{0} / \sqrt{\varepsilon_{\mathrm{rc}}+1}$.

The energy coupling to the cavity is achieved by means of a slot etched on the cavity face opposite to the slots array (middle face). The middle face is also the ground plane of the microstrip-line that feeds the whole antenna. The substrate of this microstrip-line (the lower substrate) has thickness $g$, relative permittivity $\varepsilon_{\mathrm{r}} \ell$ and loss tangent $(\tan \delta) \ell$. The size of the coupling slot is $L_{\mathrm{ac}} \times W_{\mathrm{ac}}$. These two dimensions are calculated in the same way as $L_{\mathrm{rad}}$ and $W_{\mathrm{rad}}$, but now $\lambda_{\mathrm{g}}=\sqrt{2} \lambda_{0} / \sqrt{\varepsilon_{\mathrm{rc}}+\varepsilon_{\mathrm{r} \ell}}$. In our prototype the width of the microstrip-line, $W$, is designed for a characteristic impedance of $50 \Omega$, but other impedance values are possible. Apart from its simplicity, the main advantage of this excitation is that both the antenna and the feeding structure can be fully implemented in planar technology.

Only those modes that generate surface electric current perpendicular to the coupling slot will resonate in the cavity [1]. To achieve good coupling between the feeding microstrip-line and the cavity, the former is finished with an open-circuit stub of length $L_{\text {stub }}$ beyond the coupling slot. The coupling is improved by shifting the line along the $y$ axis a distance $d_{\text {line }}$ off the slot center [10].

A metallic backplane can be placed at a distance $h$ below the microstrip-line to prevent back radiation. This distance may be 
TABLE 1 Characteristics and Dimensions of the Prototype Antenna

\begin{tabular}{|c|c|c|}
\hline \multicolumn{3}{|c|}{ Array parameters $(N \times \times M=4 \times \times 4)$} \\
\hline$d_{x}=9.60 \mathrm{~mm}$ & \multicolumn{2}{|c|}{$d_{y}=15.35 \mathrm{~mm}$} \\
\hline$L_{\mathrm{rad}}=7.52 \mathrm{~mm}$ & \multicolumn{2}{|c|}{$W_{\mathrm{rad}}=1.88 \mathrm{~mm}$} \\
\hline \multicolumn{3}{|c|}{ Cavity parameters } \\
\hline Substrate RO3003 & \multicolumn{2}{|c|}{ Resonant mode: $\mathrm{TE}_{5,0,8}$} \\
\hline$\varepsilon_{\mathrm{rc}}=3$ & \multicolumn{2}{|c|}{$a=76.77 \mathrm{~mm}$} \\
\hline $\tan \delta_{\mathrm{c}}=0.0013$ & \multicolumn{2}{|c|}{$d=76.77 \mathrm{~mm}$} \\
\hline$b=1.52 \mathrm{~mm}$ & \multicolumn{2}{|c|}{$\begin{array}{c}p_{\mathrm{p}}=1 \mathrm{~mm} \\
d_{\mathrm{p}}=0.1 \mathrm{~mm}\end{array}$} \\
\hline \multicolumn{3}{|c|}{ Feeding and coupling parameters } \\
\hline Substrate RO3010 & Line & Slot \\
\hline$\varepsilon_{\mathrm{r}} \ell_{1}=10.2$ & $W=0.58 \mathrm{~mm}$ & $L_{\mathrm{ac}}=7.42 \mathrm{~mm}$ \\
\hline $\tan \delta \ell_{1}=0.0023$ & $L_{\mathrm{stub}}=0.6 \mathrm{~mm}$ & $W_{\mathrm{ac}}=1.28 \mathrm{~mm}$ \\
\hline$g=0.64 \mathrm{~mm}$ & $d_{\text {line }}=1 \mathrm{~mm}$ & $h=7.5 \mathrm{~mm}$ \\
\hline
\end{tabular}

used to finely match the whole antenna. A good preliminary choice for this distance is a quarter free-space wavelength $[11,12]$.

As already mentioned, the principal aim of this work is to demonstrate the feasibility of the slot array in SIW technology with a planar feed. A working frequency of $10 \mathrm{GHz}$ is chosen as a good example of possible services that could incorporate this kind of antennas. The upper substrate is Rogers RO3003 of thickness $1.52 \mathrm{~mm}$. The low permittivity is to help radiation. The lower substrate is Rogers RO3010 of thickness $0.64 \mathrm{~mm}$, more convenient for the feeding microstrip-line. The electrical properties of both substrates are shown in Table 1. The SIW cavity has been designed, so that the mode $\mathrm{TE}_{5,0,8}$ resonates at $10 \mathrm{GHz}$. In our case, the election of the mode was determined by the maximum prototype size; the mode order depends on the number of alternate field maxima that can be allocated in the antenna length. However, in common applications, the number of elements in the array is determined by the specified directivity, which gives the mode order. Additional metallic posts are introduced to filter the mode $\mathrm{TE}_{7,0,6}$, which is the closest unwanted mode. These posts should be placed in those points, where the electric field associated with the desired mode is null and that of the closest unwanted mode is as high as possible.

When the radiation slots are introduced, the resonance of the desired mode shifts to lower frequencies, an expected effect observed during simulations. The more electric current lines the slots are cut off, the bigger shift of the resonance to lower frequencies. Therefore, once the slots have been drawn on the cavity, the dimensions of the entire system must be readjusted for the desired frequency of operation. Finally, a fine tuning of the most sensitive dimensions, which are mainly $L_{\text {stub }}$ and $d_{\text {line }}$, is carried out with the help of Agilent ADS software to improve the antenna performance. The optimization criterion was trading best matching at central frequency for wider frequency band. Table 1 summarizes the substrate characteristics and dimensions of our particular design. Figure 2 shows a picture of the built and measured prototype. It is worth noting that, for technological reasons, the posts defining the cavity do not satisfy the condition $p_{\mathrm{p}}$ $<4 d_{\mathrm{p}}$. However, the effects of the actual post diameter $d_{\mathrm{p}}$ have been implicitly considered, because the antenna dimensions have been optimized using a full-wave model of the SIW cavity.

\section{RESULTS}

Simulated and measured return losses of the designed antenna are compared in Figure 3. Simulations were done with the help of Agilent ADS software. The simulated $\left|S_{11}\right|$ is lower than -10 $\mathrm{dB}$ in a range of $220 \mathrm{MHz}$, which at $10 \mathrm{GHz}$ means a band-

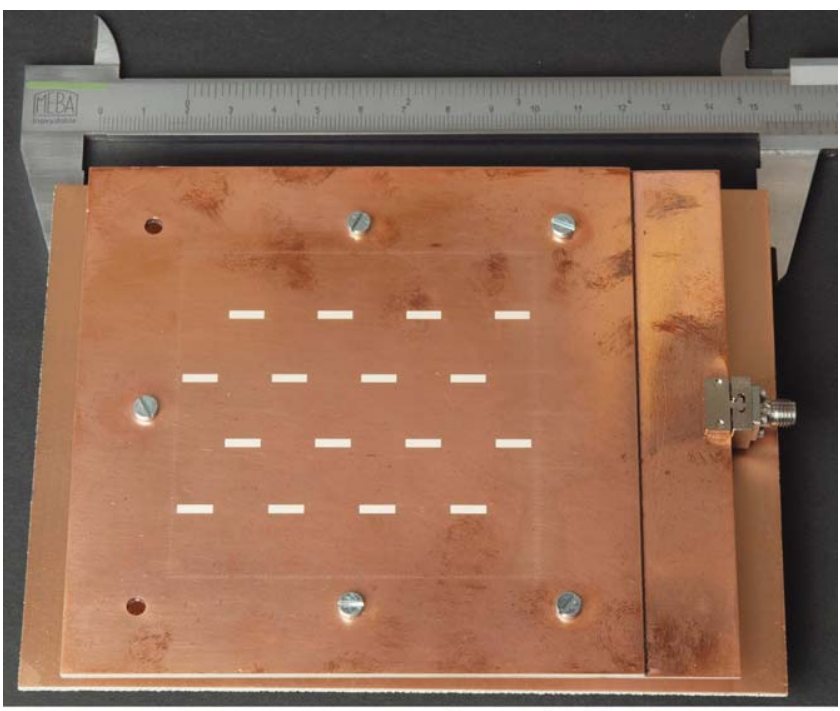

(a)

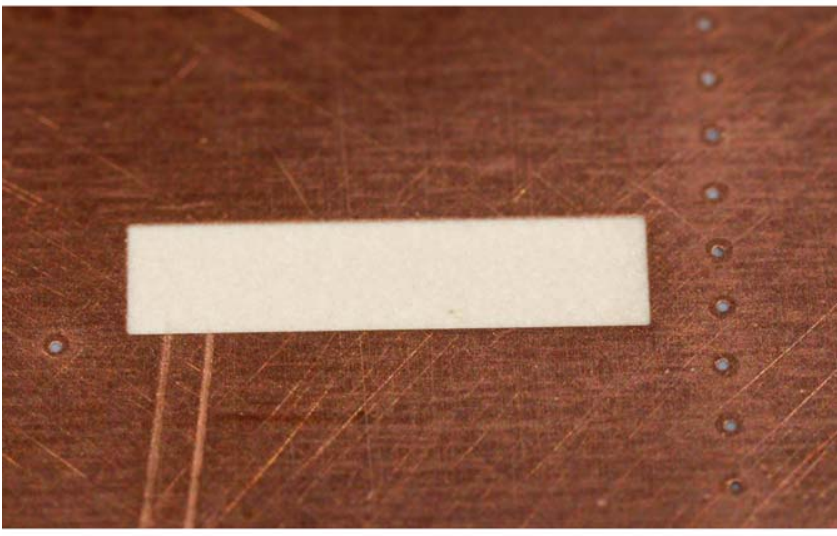

(b)

Figure 2 (a) Picture of the built and measured prototype, which includes a metallic backplane to prevent back radiation. (b) Detail of a slot and some of the via holes. [Color figure can be viewed in the online issue, which is available at wileyonlinelibrary.com]

width of $2.2 \%$. To make up for this (possible but not necessary) drawback, better matching at $10 \mathrm{GHz}$ has been traded for an increased bandwidth, as mentioned above. Taking into account

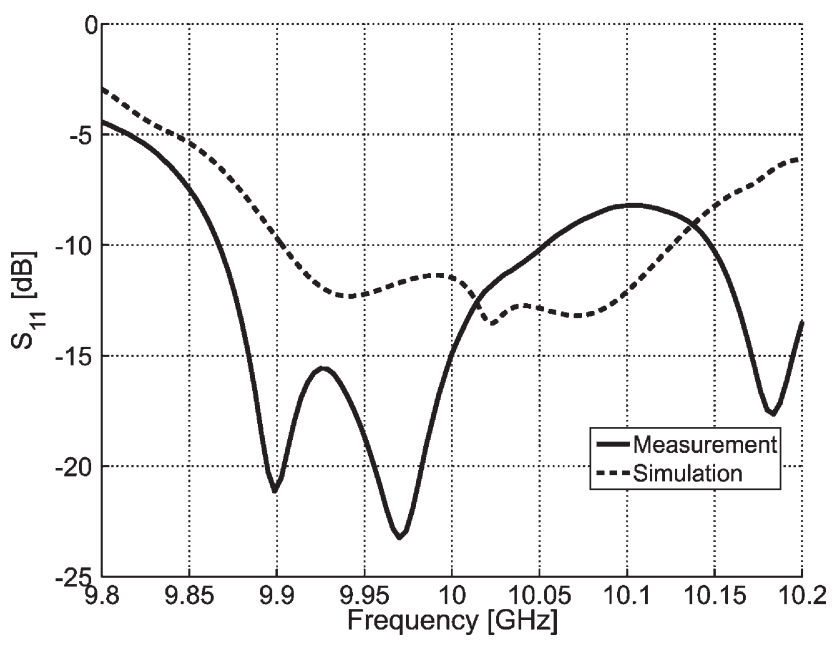

Figure 3 Absolute value of the input reflection coefficient of the antenna 


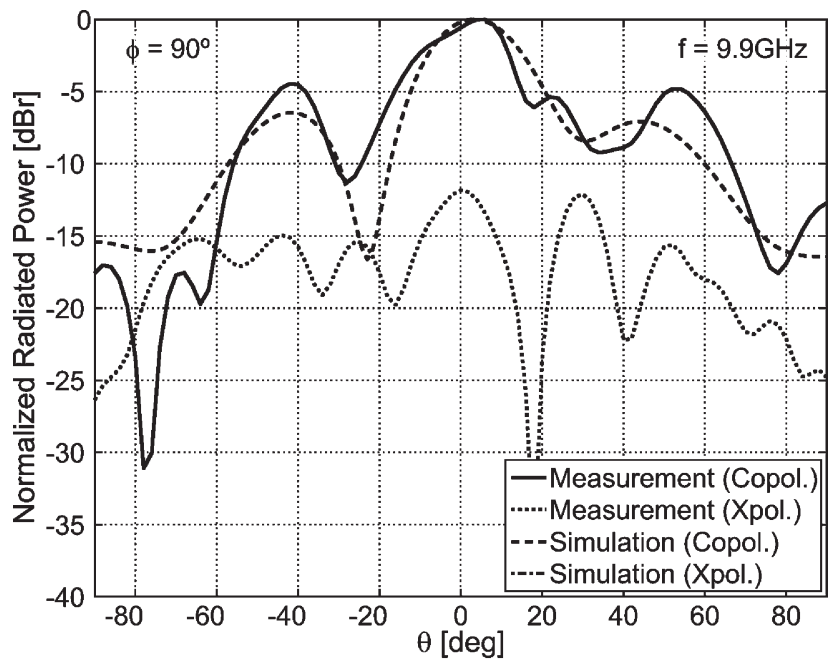

(a)

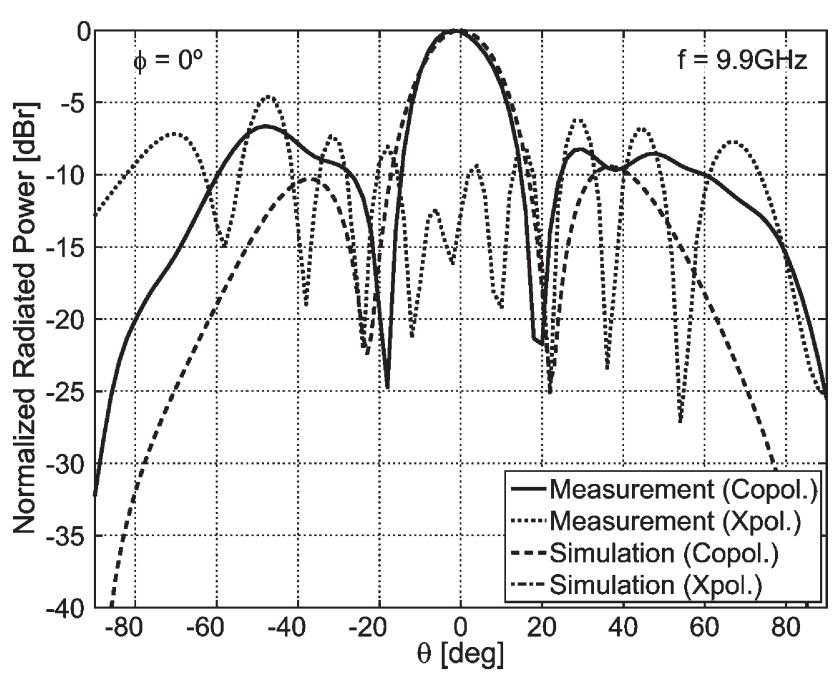

(b)

Figure 4 Simulated and measured radiation patterns at $9.9 \mathrm{GHz}$. (a) E plane. (b) H plane

the modeling limitations, a good agreement has been obtained between measured and simulated results. A shift of about 30 $\mathrm{MHz}$ (frequency error of $0.3 \%$ ) is observed in the lower cut-off frequency. The discrepancies between measured and simulated results can be attributed to the existence of undesired resonant modes in the cavity, which in practice are not completely canceled by the metallic posts.

The use of the metallic backplane to prevent back radiation could raise a spurious mode in the partially loaded parallel-plate waveguide thus formed. At high frequency, which is the case of our antenna, the mode is independent of the separation $h+g$ [13]. In our case $h=7.5 \mathrm{~mm}$, whereas $g=0.64 \mathrm{~mm}$, and then, we can consider that the antenna feeding circuit behaves as if the backplane was not present. Of course, the surface wave in the microstrip substrate could still propagate along the dielectric-air interface. That mode would be the fundamental transverse magnetic (TM) mode, which has a zero cut-off frequency. There are two possibilities to excite that mode. The first option is coupling from the quasi-TEM mode in the microstrip-line. According to Ref. 14, the frequency, at which significant coupling occurs between the quasi-TEM mode and the lowest order surface wave mode, can be calculated in our substrate to be 42.2 $\mathrm{GHz}$, far beyond the operating frequency. The second option is that the coupling slot excites the TM mode. In this case, although the magnetic field in the coupling slot is transversal, the longitudinal electric field in the slot is short circuited by the surrounding metallic plane, that is, the coupling slot does not favor the development of the fundamental TM mode.

Figures 4-6 show simulated and measured radiation patterns in $\mathrm{E}$ and $\mathrm{H}$ planes (planes $y-z$ and $x-z$, respectively, in Fig. 1) at three different frequencies. There is a fair agreement between simulated and measured results, with the only exception of the crosspolar level. Although the simulated crosspolar level is almost zero, the corresponding measured level is not negligible, presumably due to imperfections in manufacturing, radiation from the side walls of the cavity, and the existence of undesired resonant modes in the cavity. The pure modal distribution obtained in simulation is probably not achieved in the actual cavity. Other than this, the patterns are consistent with what could be expected of a $4 \times 4$ array. Uniform illumination of the array elements is not perfectly achieved in every element of the array; this is the price paid for the absence of a feeding network.

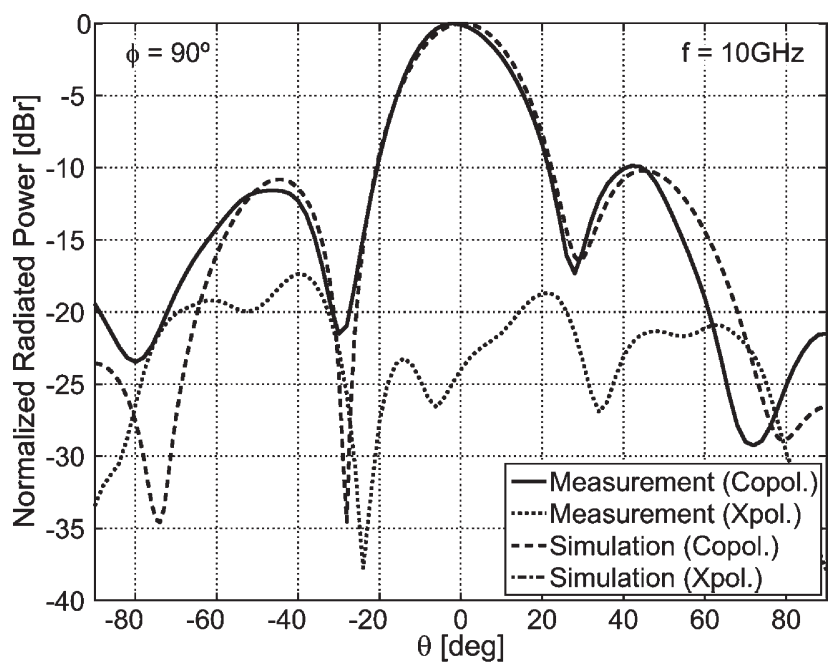

(a)

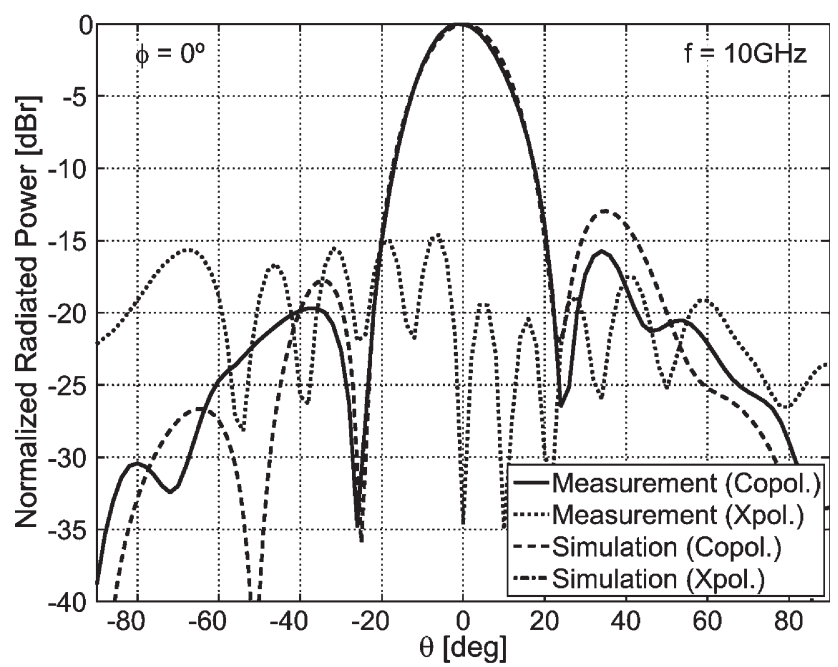

(b)

Figure 5 Simulated and measured radiation patterns at $10.0 \mathrm{GHz}$. (a) E plane. (b) H plane 


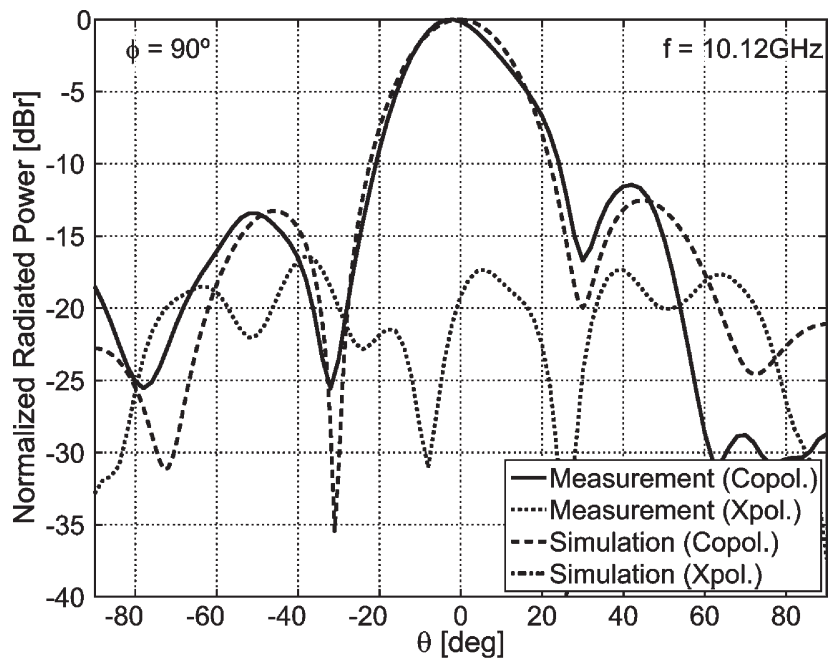

(a)

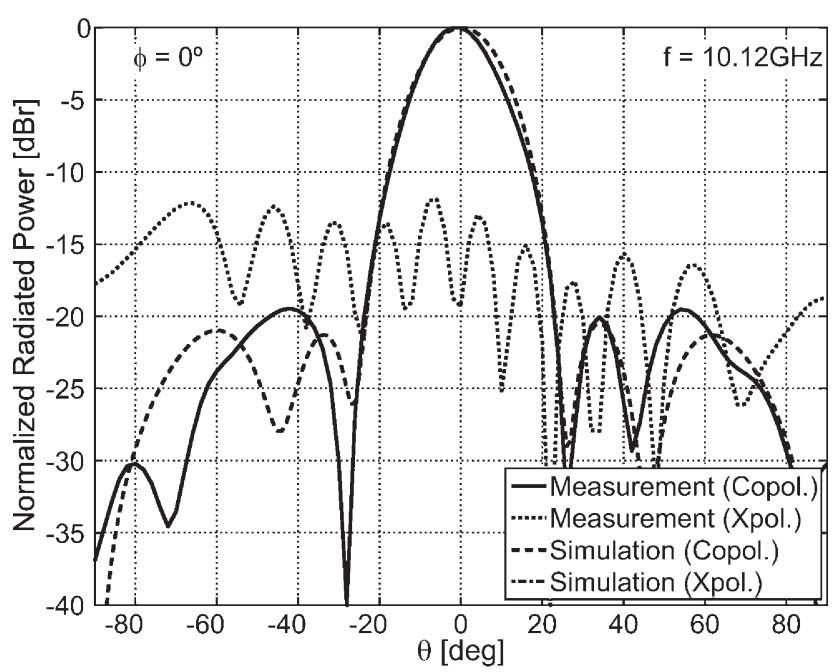

(b)

Figure 6 Simulated and measured radiation patterns at $10.12 \mathrm{GHz}$. (a) E plane. (b) H plane

The measured antenna gain, which amounts up to $13 \mathrm{~dB}$ at $10 \mathrm{GHz}$, is compared to the simulated value in Figure 7. Theoretical prediction of the directivity of a $4 \times 4$ array with uniform illumination and separation between elements 9.6 and $15.35 \mathrm{~mm}$, respectively, is about $15.2 \mathrm{~dB}$ at $10 \mathrm{GHz}$. If the element directivity is about $2.1 \mathrm{~dB}$ and the expected efficiency (due to RO30XX losses) at $10 \mathrm{GHz}$ is estimated to be $-4 \mathrm{~dB}$, the gain would be near $13 \mathrm{~dB}$. This value confirms that only a negligible fraction of the antenna input power is not coupled from the input line to the cavity.

The gain level, together with the overall good agreement between simulation results and measurements observed so far, is considered as a successful assessment of the proposed antenna concept as well as its theoretical modeling.

\section{CONCLUSIONS}

A slot array etched on the upper face of a SIW cavity has been demonstrated to be a convenient antenna solution for wireless applications. The cavity is fed by a microstrip-line aperture coupled by means of a slot etched in the opposite face of the cavity. The simplicity of the feeding line is largely worth the

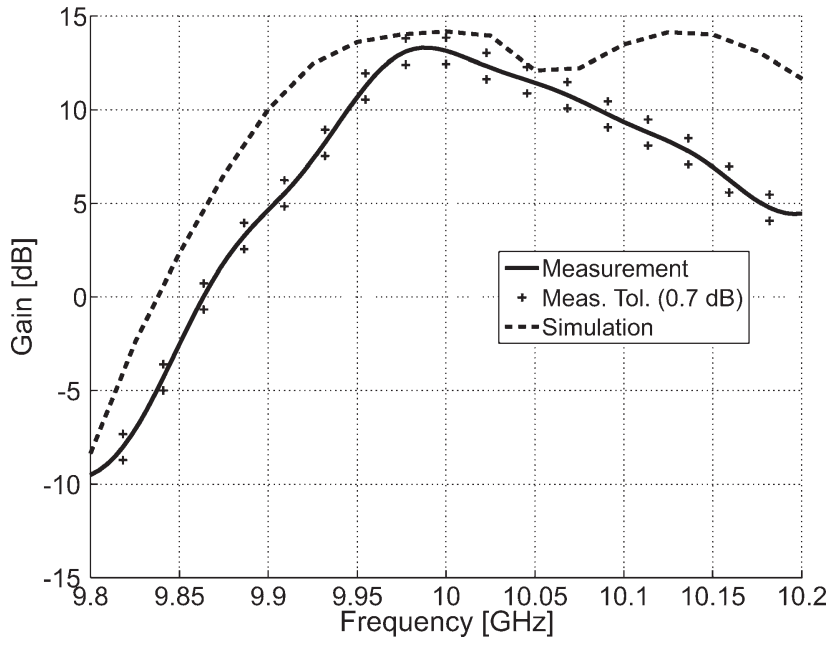

Figure 7 Simulated and measured gain

effort of designing the cavity and the slot locations. The antenna thickness is $0.33 \times \lambda_{0}$, backplane included, and can be entirely manufactured with conventional low-cost printed-circuit technology. The antenna performance in terms of matching, bandwidth, and radiation patterns is comparable to that of more complicated designs, the only drawback being its relatively narrow frequency bandwidth.

\section{REFERENCES}

1. R.S. Elliott, Antenna theory and design, Prentice Hall, New York, NY, 1981

2. F.J. Paoloni, A cavity-backed resonant slot array-Theory and measurement, IEEE Trans Antennas Propag 28 (1980), 259-263.

3. S.V. Savov and H.D. Hristov, Cavity-backed slot array analysis, IEE Proc H-Microwaves Antennas Propag 134 (1987), 280-284.

4. A. Kumar and H.D. Hristov, Microwave cavity antennas, Artech House, Norwood, MA, 1989.

5. G.Q. Luo, Z.F. Hu, L.X. Dong, L.L. Sun, Planar slot antenna backed by substrate integrated waveguide cavity, IEEE Antennas Wireless Propag Lett 7 (2008), 236-239.

6. L. Yan, W. Hong, G. Hua, J. Cheng, K. Wu, and T.J. Cui, Simulation and experiment on SIW slot array antennas, IEEE Microwave Wireless Components Lett 14 (2004), 446-448.

7. K. Wu, D. Deslandes, and Y. Cassivi, The substrate integrated circuits-A new concept for high-frequency electronics and optoelectronics, In: 6th international conf telecomm modern satellite, Cable broadcast services TELSIKS'03, Yugoslavia, 2003, pp. 3-10.

8. D. Deslandes and K. Wu, Single-substrate integration technique of planar circuits and waveguide filters, IEEE Trans Microwave Theory Tech 51 (2003), 593-596.

9. Y. Cassivi, L. Perregrini, P. Arcioni, M. Bresan, K. Wu, and G. Conciauro, Dispersion characteristics of substrate integrated planar waveguide, IEEE Microwave Wireless Components Lett 12 (2002), 333-335.

10. R. Garg, P. Bhartia, I. Bahl, and A. Ittipiboon, Microstrip antenna design handbook, Artech House, Norwood, MA, 2001.

11. J.-F. Zürcher and F.E. Gardiol, Broadband patch antennas, Artech House, Norwood, MA, 1995.

12. Y. Yoshimura, A microstripline slot antenna, IEEE Trans Microwave Theory Tech 20 (1972), 760-762.

13. R.E. Collin, Foundations for microwave engineering, 2nd ed., McGraw-Hill, New York, NY, 1992, pp. 117-125.

14. K.C. Gupta, R. Garg, I.J. Bahl, and P. Bhartia, Microstrip lines and slotlines, 2nd ed., Artech, Norwood, MA, 1996, p. 112

(C) 2011 Wiley Periodicals, Inc. 